\title{
Metodología de la investigación. Las prácticas docentes de los profesores de estocástica en ingeniería
}

\author{
Methodology of The Investigation. The Training of Teachers Stochastic In The Faculty \\ Of Engineering
}

Pedro Rocha Salamanca

Universidad Distrital Francisco José de Caldas- Bogotá - Colombia. pgrocha@udistrital.edu.co

\section{Resumen}

Este trabajo es una aproximación metodológica, que permite construir la trayectoria docente de los profesores de ingeniería cuando enseñan estadística y probabilidad en las Facultades de Ingeniería.

\section{Abstrac}

This paper is a methodological approach that allows teachers to construct the trajectory of the engineering faculty when they teach statistics and probability in the Faculty of Engineering.

\section{Palabras claves}

Probabilidad, estadística, práctica docente, etnografía, Educación en ingeniería, metodología.

\section{Keywords}

Probability, statistics, teaching practice, ethnography, engineering education, methodology.

\section{Introducción}

Las investigaciones en el campo educativo y espećficamente en el área de la educación estocástica, necesariamente tiene que dedicar tiempo importante a la indagación, búsqueda y análisis de la metodología a ser implementada para el desarrollo de la investigación, mostrando el proceso de construcción de las bases teóricas del diseño metodológico, en este caso las practicas docentes de los profesores de Ingeniería, lo que permite igualmente seguir construyendo aportes teóricos y metodológicos importantes a otras propuestas similares. Partimos entonces definiendo que el presente diseño metodológico y en general toda la investigación, se basa en efectuar una reformulación constante de los aspectos teóricos, metodológicos, de validez, etc. como consecuencia natural al incluir los nuevos resultados dentro del trabajo

Esta propuesta metodológica se enmarca dentro del campo de investigación denominado Educación Estocástica (rocha, 2007) particularmente se ubica como un trabajo doctoral enfocado principalmente a los aspectos didácticos que permiten el análisis y comprensión de cómo ha sido el desarrollo de las prácticas docentes de los profesores de Ingeniería cuando se han enfrentado al problema de enseñar probabilidad y estadística, como son actualmente y termina con una propuesta de modelo de práctica que puede ser implementada por los profesores de estocástica en las facultades de ingeniería 
El diseño metodológico se encuentra enmarcado dentro del desarrollo de tres fases interdependientes. Para cada una de las fases diseñadas se utilizarán diferentes métodos de recolección de la información dependiendo de los objetivos a lograr. Por tanto, es necesario determinar cada uno de los momentos que conforman la respectiva fase.

> El primero denominado preparatorio; ayyo objetivo principal es elegir un marco teórico que permita la obtención de los objetivos propuestos en cada una de las fases.

$>$ El segundo momento denominado construcción teórico general, el cual se enmarca en el estudio y selección de las metodologías de investigación etnográficas para la realización de la indagación documental, entrevistas, grabaciones y encuestas necesarias para recolectar la información. De igual manera se escogerán los métodos estadísticos adecuados para el análisis de la información.

$>$ El tercero denominado recopilación de la información. Se trabajará en el diseño, planeación, ejecución y validación de los instrumentos necesarios para recolectar la información. La aplicación de instrumentos de recolección de información y registros para realizar la descripción del cambio de las prácticas, sus factores determinantes y consecuencias se realizará en espacios de tiempo en puntos o periodos especificados,

$>$ El cuarto, sistematización de la información, análisis de instrumentos de observación, validación de los resultados, triangulación.

D El quinto, análisis de los resultados obtenidos elaboración de reporte en términos de los propósitos teóricos de la investigación

\section{Perspectiva metodológica para el estudio de las prácticas docentes de los profesores de ingeniería}

En contraste con los métodos tradicionales de investigación, durante las últimas décadas los investigadores en didáctica han recurnido a los métodos que desarrolla la investigación etnográfica, ya que por sus características permite dar una mejor explicación a los problemas planteados por la comunidad académica intemacional. De acuerdo con esta postura, la perspectiva metodológica que sustenta el estudio e interpretación de la interacciones del profesor de ingeniería con el saber y sus prácticas docentes se desarrolla a partir de la concepción teórica denominada investigación etnográfica, (Goetz, J. y LeCompte, M., 1988) la aual presenta las siguientes características enfocadas dentro del contexto de la presente tesis:

> La realización de una descripción pormenorizada de las acciones del profesor de ingeniería en tres momentos diferentes de su practica docente a partir del estudio de las funciones y actividades desarrolladas cuando diseñan sus dases, cuando realizan la gestión de éstas en los diferentes espacios de formación y cuando evalúan el proceso de aprendizaje de los estudiantes.

$>$ La validación constante del proceso de recopilación de información con el propósito fundamental de establecer si es necesario recolectar nuevos datos que permitan mejorar las interpretaciones ganando así en la comprensión y análisis de la trayectoria docente.

> A partir de la información recolectada, se construye una trayectoria denominada docente que es de tipo emergente, es decir, a partir del análisis, la comprensión e interpretación de las acciones del profesor en los diferentes espacios de formación se establecen secuencias, que son recurrentes en el tiempo, que conforman episodios, por lo tanto, se pueden agrupar en un modelo de profesor que presenta regularidades, pero además, con la indusión de nueva información puede ser reformulado y por tanto refinado

> La comprensión de las interrelaciones observables a la trayectoria docente y epistémica se sustenta en los significados que le otorguen los profesores y la interpretación del investigador. 
Por tanto, se asignará importancia crucial a las explicaciones entregadas por los docentes investigados, con especial énfasis en las justificaciones que le otorgan a cada uno de los episodios, es decir a lo que se denomina en etnometodología las prácticas explicativas de los profesores

> A medida que se construye la trayectoria docente pueden ser reformuladas las hipótesis, el objetivo principal no es comprobar las hipótesis propuestas al iniciar el trabajo doctoral, sino más bien validarlas.

\section{FASE I}

La educación estocástica y su historia, desde las prácticas docentes

Los objetivos principales de esta fase están encaminados a indagar principalmente sobre dos problemas que permiten contestar a la pregunta de investigación: ¿Cuáles fueron las prácticas docentes predominantes en las Facultades de Ingeniería por parte de los profesores, cuando se enfrentaron al problema de enseñar objetos de estudio estocásticos?

\section{Momento preparatorio}

\section{Definición y delimitación de los polos a ser estudiados}

La investigación retomaría el Tetraedro didáctico como una herramienta de investigación para el análisis de los procesos de enseñanza - aprendizaje en el aula de clase, y que se constituińa en la representación del sistema didáctico (estudiante, saber, profesor y entomo) puesto que, permite visualizar, comprender y representar los componentes y las relaciones que se establecen entre los mismos además de definir el lado correspondiente al que esta investigación hace énfasis, señalando que lo relevante, es analizar las interrelaciones entre el polo didáctico, diseño de las actividades por parte del profesor, y el polo epistémico

\section{Construcción de los instrumentos}

Otro elemento fundamental, está relacionado con el diseño de instrumentos de indagación que se refiere a la utilización de técnicas para la recolección de información, entendida según JeanMarie de Ketele (1995) como "un proceso que induye la atención voluntaria y la inteligencia, orientadas por un objeto para obtener de él información. "Dentro de las siguientes características:

D Se privilegia el estudio de las acciones del profesor en tres estados interconectados denominados como diseño, gestión y evaluación en un espacio temporal determinado

$>$ Se reconoce a manera de hipótesis que el lugar privilegiado para la observación de la ruta docente es el aula de dase universitaria. Lo cual implica, que para entender el significado de los hechos que ocurren en el momento de abordar los objetos de estudio estocasticos, se realice desde una perspectiva compleja y dinamica lo que afecta el tratamiento y análisis de los datos.

$>$ Por la naturaleza de la investigación se estudia principalmente las interacciones entre los diferentes actores que intervienen en la manifestación de la trayectoria; los estudiantes, los profesores y el entomo, elementos a tener en cuenta al describir, comprender, analizar y construir la ruta docente más probable.

El primer problema se encuentra enmarcado dentro de la descripción, análisis y comprensión de las causas que permitieron la inserción en las facultades de ingeniería de los objetos de estudio estocásticos, altemamente con el estudio de cómo han venido evolucionando los objetos de estudio estocásticos involucrados en la enseñanza de la probabilidad y estadística en las facultades de Ingeniería.

En el aumplimiento de los objetivos propuestos en la fase I se decidió entonces utilizar los siguientes instrumentos de indagación: 
> Revisión documental de algunos planes de estudio utilizados en las últimas décadas en las facultades de ingeniería.

D Estudio histórico de algunos libros de texto de probabilidad y estadística.

$>$ El segundo problema se ubica en la descripción de como han evolucionado las diferentes funciones que realizan los profesores en relación al diseño, ejecución y evaluación del proceso de enseñanza aprendizaje, los instrumentos a implementar serían:

$>$ Revisión documental de algunos programas de los profesores en las dases de probabilidad y estadística.

D Entrevistas a expertos, profesores e historiadores.

\section{Momento construcción teórico general}

Métodos de recolección de la información

Uno de los métodos más utilizados por los investigadores en educación cuando se utiliza la entrevista como instrumento de recolección de información es la denominada Teoría fundamentada en los datos de Glaser y Strauss.

Según Vasilachis de Gialdino (2006) "La metodología propuesta por la teoría fundamentada se basa en dos grandes estrategias: el método de la comparación constante, y el muestreo teórico. $A$ través del método de la comparación constante el investigador recoge, codifica y analiza datos en forma simultánea, para generar teoría. Es decir, estas tareas no se realizan en forma sucesiva sino simultanea, y no están dirigidas a verificar teorías, sino a demostrar que son plausibles. Esto a su vez, se realiza a través de dos procedimientos:

> Ajuste: las categorías deben surgir de los datos y deben ser fácilmente aplicables a ellos; y

> Funcionamiento: deben ser significativamente apropiadas y capaces de explicar la conducta en estudio." (Pág. 155)

Procedimiento para la realización de las entrevistas.

$>$ Objetivo

Elaborar una descripción detallada a partir de los relatos de los profesores de las formas en que realizaban las dases durante la segunda mitad del siglo $\mathrm{XX}$, a partir de la identificación de los acontecimientos o episodios significativos dentro del aula caracterizando las acciones que generan la trayectoria o ruta docente.

$>$ Población objetivo

Profesores de Estocástica en las facultades de Ingeniería durante los ochentas 0 antes.

Por ser ésta una población muy específica se realizará la selección por conveniencia de profesores que hayan sido docentes en las facultades de Ingeniería, si es posible en los espacios relacionados con la probabilidad y estadística.

$>$ Diseño del muestreo

Para esta fase se utiliza como técnica de muestreo el denominado teórico que tienen como característica primordial la escogencia de los entrevistados tomando como criterio principal el aporte a la elaboración de una teoría que describa como se desarrollaban las dases de probabilidad y estadística durante la segunda mitad del siglo $X X$, cuál era la estructura y como se integró el conocimiento estocástico en las Facultades de Ingeniería.

\section{Momento recopilación de la información}

La fuente principal de información como se ha presentado es la entrevista, pero también se induirán otras fuentes de segundo nivel como; documentos históricos, libros de texto, y el 
resultado de otras investigaciones que no tenían como finalidad principal el estudio de las prácticas docentes de los profesores universitarios.

\section{FASE II}

Las prácticas docentes actuales

Después de conocer la historia y evolución de las prácticas docentes, es necesario para el desarrollo de la propuesta determinar, cómo son actualmente las prácticas docentes de los profesores de estocástica en las Facultades de Ingeniería, es decir, determinar qué enfoques didácticos caracterizan las prácticas de los profesores. El resultado final es una descripción interpretativa, correlacional y reconstructiva del estado actual de las formas teórico prácticas que ponen en juego los profesores, así como la comprensión del estado actual de la didáctica de la estocástica, posibilitando entonces una construcción teórica que tiene como sustento una perspectiva general de cómo han evolucionado las practicas docentes en las facultades de ingeniería.

Momento preparatorio

En esta fase, se utilizarán dos tipos de métodos para la recolección de la información el primero agrupa las entrevistas personales y encuestas autoaplicadas para los cuales existe un diseño a partir de la teoria general del muestreo, el segundo agrupa el estudio de documentos y la grabacion de algunas sesiones de dase que por su naturaleza determinan otro tipo de tratamiento para el analisis y sitematizacion de resultados.

Diseño de la encuesta por muestreo.

Si se desea realizar una delimitación de la población a estudiar tendríamos que referimos a induir en ella a los profesores de estadística de las universidades públicas en la ciudad de Bogotá que enseñan o son responsables de la enseñanza de la probabilidad y estadística en las facultades de Ingeniería.

En el aumplimiento de los objetivos propuestos se resolvió entonces utilizar los siguientes instrumentos de indagación:

1. Encuestas a los docentes de ingeniería donde se pueda reconstruir de forma compleja el sistema de prácticas en el aula de dase, indagando sobre las principales acciones que realizan cuando diseñan, desarrollan y evalúan los programas de los diferentes espacios de formación.

2. Entrevistas a un conjunto de informadores expertos, para describir cómo se han venido induyendo dentro de los currículos los objetos de estudio estocásticos en los programas de ingeniería.

3. Estudio de documentos para los cuales se realizarán observaciones sistemáticas de los instrumentos de evaluación de los docentes, con el propósito de validar la ruta docente emergente.

4. Grabación de algunas sesiones de dase de los docentes, para triangular información

\section{Momento construcción teórico general}

Métodos de recolección de la información

Encuestas a docentes.

Instrumento desarrollo general de la clase. Perspectiva del profesor

$>$ Objetivo general

Determinar auáles son los principales factores que inciden en el diseño, gestión y evaluación de los espacios de formación por parte de los profesores de probabilidad y estadística de las facultades de ingeniería. 


\section{Población objetivo}

Profesores de estadística de las universidades públicas en la ciudad de Bogota que enseñan o son responsables de la enseñanza de la probabilidad y estadística en las facultades de Ingeniería.

$>$ Diseño de muestreo

$>$ Análisis de datos.

Para realizar la descripción de los factores que afectan las acciones del profesor en el proceso de diseño gestión y evaluación se utilizará información de tipo multivariado que es resultado de observar para un conjunto de docentes una serie de respuestas, por tanto la matriz inicial de datos tiene la siguiente estructura:

\begin{tabular}{|c|c|c|c|c|}
\hline Profesor & Pregunta 1 & Pregunta 2 & $\ldots$ & Pregunta k \\
\hline Profesor 1 & $X_{1,1}$ & $X_{1,2}$ & & $X_{1, k}$ \\
\hline Profesor 2 & $x_{2,1}$ & $x_{2,2}$ & & $x_{2, k}$ \\
\hline : & . & : & & \\
\hline i & & & & \\
\hline Profesor $n$ & $x_{n, 1}$ & $X_{n, 2}$ & & $X_{n, k}$ \\
\hline
\end{tabular}

La observación $X_{i, j}$ será la respuesta que entrega el profesor i-ésimo a la pregunta j-ésima, la escala de medida para este tipo de instrumentos serán aquellas que reflejan una cantidad relativa a la medición de la importancia que le otorga a cada una de las acciones que realiza en los diferentes espacios de formación cuando enseña probabilidad o estadística. La ventaja de este tipo de escala tiene que ver con la característica que tienen un patrón de medida que permite realizar interpretaciones significativas dentro del análisis de los datos.

\section{Momento recopilación de la información}

Instrumento practicas docentes facultad de ingeniería

Desarrollo general de la clase. Perspectiva del profesor

El diseñar un instrumento que permita conocer las acciones del profesor sobre diferentes acontecimientos significativos referidos a su trabajo dentro y fuera del aula, a partir de los siguientes aspectos: polo epistémico y polo didáctico

Objetivo general:

Desarrollar una descripción pormenorizada de la clase realizada (u observada) que permita la identificación de los acontecimientos significativos del aula; y análisis de la dinámica del hecho educativo, caracterizando las acciones que generan la ruta docente.

Objetivos específicos:

$>$ Indagar como se han induido los objetos de estudio estocásticos en el aula de ingeniería a través del tiempo.

> Describir, enfocar y analizar la descripción general desde cada uno de los polos, identificando las acciones realizadas, la influencia en las interacciones, y en el cambio de manifestaciones conducta cognitiva dadas en el aula

$>$ Identificar patrones de interacción y acciones significativas de los estudiantes que les permite generar conocimiento.

\section{Instrumentos a utilizar}

En relación con el diseño de las clases

Califique según la importancia de uno a cinco siendo uno la menor valoración, las siguientes actividades que pueden ser realizadas antes de las sesiones de dase. 
Preparar material de tipo teórico que pueda servir de apoyo a los estudiantes en las clases Seleccionar ejercicios de libros de texto para que sean desarrollados por los estudiantes Construir presentaciones, del tipo Power Point para exponerlas en las sesiones de clase Construir situaciones fundamentales para que sean solucionadas por los estudiantes. Diseñar proyectos para que los estudiantes los desarrollen a lo largo del semestre Diseñar talleres con las temáticas a desarrollar en las sesiones de dase por los estudiantes durante el semestre

En relación con la implementación de las sesiones de clase

Califique según la importancia de uno a cinco siendo uno la menor valoración, las siguientes actividades que pueden ser realizadas durante las sesiones de dase.

\begin{tabular}{|l|l|}
\hline \multicolumn{1}{|c|}{ Actividad } & Calificación \\
\hline Desarrollar ejercicios en el tablero & \\
\hline Presentar conceptos & \\
\hline Estudio de casos & \\
\hline Trabajo en grupo de los estudiantes & \\
\hline Exposiciones de los estudiantes & \\
\hline Ejercicios fuera de dase & \\
\hline Problemas de aplicación & \\
\hline Discusión solución de problemas por los estudiantes & \\
\hline Ejercicios de libros para solucionar & \\
\hline Explicación de teorías & \\
\hline Formulación de preguntas a los estudiantes & \\
\hline
\end{tabular}

En relación con la evaluación

Califique según la importancia de uno a cinco siendo uno la menor valoración, las siguientes que son utilizadas por los docentes para evaluar el proceso de enseñanza.

\begin{tabular}{|l|l|}
\hline \multicolumn{1}{|c|}{ Actividad } & Calificación \\
\hline Talleres desarrollados en la clase & \\
\hline Parciales escritos & \\
\hline Ejercicios de libros para solucionar & \\
\hline Proyectos de trabajo-aula & \\
\hline Ensayos & \\
\hline Talleres desarrollados en la clase & \\
\hline Exposiciones de los estudiantes & \\
\hline
\end{tabular}

\section{Resultados esperados}

Con la construcción metodológica presentada se espera poner en práctica un modelo de trabajo para determinar cómo han sido y cómo son las practicas de los docentes en las facultades de ingeniería.

\section{Bibliografía}

De ketele, J. y Rogers, C. (1995). Metodología para la recogida de información. Madrid,

España. Ed. española: Editorial La Muralla, S. A. Goetz, J. y LeCompte, M. (1988). Etnografía y diseño cualitativo de investigación educativa. Morata, Madrid, España.

Rocha P., (2007). Cuadernos de investigación. No. 10. Educación Estocástica. Bogotá, Colombia: Universidad Distrital "Francisco José de Caldas". 
Tecné, Episteme y Didaxis: TE $\Delta$ No. Extraordinario, 2009

$4^{\circ}$ Congreso Internacional sobre Formación de Profesores de Ciencias

Vasilachis de Gialdino (2006) Estrategias de investigación cualitativa, Editorial Gedisa, Barcelona España. 